\title{
Comments on Simplified Treatment of Intrabeam Scattering
}

\author{
A. V. Fedotov
}

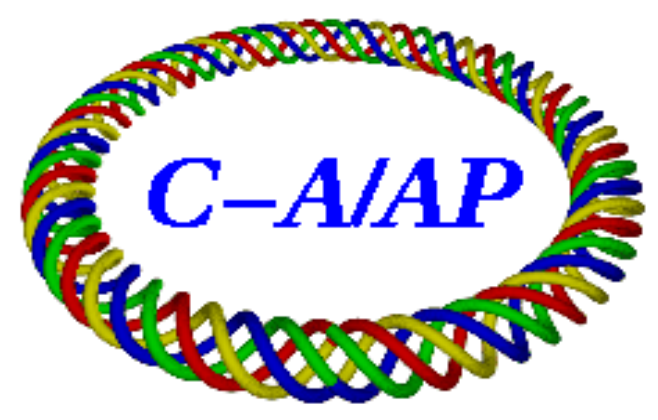

Collider-Accelerator Department Brookhaven National Laboratory Upton, NY 11973 


\title{
Comments on simplified treatment of intrabeam scattering using plasma approach.
}

\author{
A.V. Fedotov
}

(September 16, 2004)

\begin{abstract}
The theory of multiple small-angle Coulomb scattering in circular accelerators is well developed. It is typically called the Intrabeam Scattering (IBS). Similar multiple small-angle Coulomb scattering was long been developed in plasma physics (corresponding growth rate will be referred here as "gas-relaxation" formula). The major purpose of high-energy electron cooling in RHIC [1] is to overcome emittance growth due to the IBS and significantly increase an average integrated luminosity in the collider. For studies of the RHIC-II upgrade, two computer codes, which describe an ion beam dynamics as a result of cooling, IBS and other effects, are presently under development: the BetaCool [2] and the SimCool [3] codes. While the BetaCool code calculates the IBS using standard formulas which take into account real lattice of the accelerator, the original version of the SimCool code was based on an approximate treatment of multiple small-angle Coulomb scattering based on the plasma approach. The purpose of this note was to explore applicability of such an approach for the high-energy case of RHIC and compare this approach with standard IBS treatment. In the course of this study it was found that a good review of similar topic already exists [4], so that we use results of the review, repeating some major points here, for convenience.
\end{abstract}




\section{GAS RELAXATION FORMULAS}

Diffusion coefficients for a spatially uniform gas of density $n$ and isotropic Maxwellian distribution are well known. The rate of such diffusion can be written as

$$
\tau^{-1}=\frac{4 \pi n(Z e)^{4}}{m^{2}} \frac{\Lambda}{\Delta^{3}}
$$

where $\Delta$ is the one-dimensional $\mathrm{rms}$ velocity and $\Lambda$ is the Coulomb logarithm. Equation ( 1 ) shows that the heating rate is determined by the 6-D phase-space density of the gas

$\mu=n /\left(m^{3} \Delta^{3}\right)$, where $n$ is the spatial density. Similarly, the diffusion coefficients can be derived for a longitudinally collapsed velocity distribution ("flat distribution"):

$$
f(\bar{r}, \bar{v})=\frac{n}{\pi \sqrt{2 \pi} \Delta_{\perp}^{2} \Delta_{\|}} \exp ^{-\left(v_{x}^{2}+v_{y}^{2}\right) / \Delta_{\perp}^{2}} \exp ^{-v_{z}^{2} /\left(2 \Delta_{\|}^{2}\right)},
$$

with $\Delta_{\|} \ll \Delta_{\perp}$. The resulting diffusion coefficient is [4]:

$$
D_{z z}=\frac{4 \pi n(Z e)^{4}}{m^{2}} \frac{\Lambda}{\Delta_{\perp}}\left[\sqrt{\pi} \exp ^{-u^{2} /\left(2 \Delta_{\perp}^{2}\right)} I_{0}\left(\frac{u^{2}}{2 \Delta_{\perp}^{2}}\right)\right] .
$$

Due to a slow decrease of function in the square brackets with its argument, one can replace expression in the square brackets by 1 . As a result, one gets

$$
D_{z z} \approx \frac{4 \pi n(Z e)^{4}}{m^{2}} \frac{\Lambda}{\Delta_{\perp}}
$$

The growth rate in the longitudinal directions is then given by

$$
\tau_{\|}^{-1}=\frac{1}{\Delta_{\|}^{2}} \frac{d \bar{v}_{z}^{2}}{d t}=4 \pi m(Z e)^{4} \mu \Lambda \frac{\Delta_{\perp}}{\Delta_{\|}},
$$

where the 6-D phase-space density $\mu$ is defined as $\mu=n /\left(m^{3} \Delta_{\perp}^{2} \Delta_{\|}\right)$.

\section{INTRABEAM SCATTERING}

When particles in a beam scatter within each other one needs to consider both large and small angle scattering. The effect when particles can be lost as a result of a single collision event (large-angle scattering) is called Touschek effect [5]. When the scattering angles are 
small, random addition of such small scattering events can lead to a growth of beam dimensions. Such a multiple Coulomb scattering was first applied to explain emittance growth in electron beams (Bruck, Le Duff [6]) and was called "multiple Touschek effect". Typically, in electron machines the longitudinal beam temperature is much smaller than the transverse, an assumption which was used in original studies of the Touschek effect. Multiple Coulomb scattering was later generalized by Piwinski [7] for proton machines without making any restrictions on the magnitude of beam temperatures, thus making it possible to transfer energy from the longitudinal into transverse via collisions. The generalized treatment of multiple small-angle Coulomb scattering was then renamed as the Intrabeam Scattering (IBS). The IBS theory was later extended to include variations of the betatron functions and momentum dispersion function along the lattice, and was summarized by Martini (typically referred to as Martini's model) [8]. Another approach to IBS using the scattering matrix formalism from quantum electrodynamics was used by Bjorken and Mtingwa (B-M model) [9]. Both B-M and Martini's models are in good agreement with one another. In what way IBS in particle beams is different from similar scattering of gas molecules? In circular accelerators, the curvature of the orbit produces a dispersion. Because of the dispersion, a change of energy leads to change in the betatron amplitude. In other words, we have coupling of the longitudinal and transverse motion. Another consequence of this curvature effect is the negative-mass behavior of particles so that conservation law of beam temperature leads to a simple conclusion that below transition energy one can have an equilibrium between the transverse and longitudinal temperatures while above transition (in the "negative-mass" regime) there is a continuous emittance increase in both transverse and longitudinal directions. Is this coupling effect always important in circular accelerator and one always needs to use standard IBS approach rather than the gas-relaxation formula? This question was studied in detail by Sorensen [4]. He found that the IBS growth rates can be simply represented by an universal curve. When plotted against parameter $\epsilon /(\Delta p / p)^{2}$ (where $\epsilon$ in the rms beam emittance) such an universal curve shows a minimum ("brake-up") point. To the left of such a minimum the transverse growth rate dominates, while to the right - the growth 
rate is dominated by the longitudinal growth rate. This was explained by a collapse of the longitudinal velocity distribution with energy. The longitudinal and transverse velocities spread in the beam rest frame are given by:

$$
\Delta v_{\|}=\frac{\Delta p}{\gamma m}, \quad \Delta v_{x}=\frac{1}{m} \sqrt{\frac{\epsilon}{\beta_{x}}} p,
$$

which gives

$$
\frac{\epsilon}{(\Delta p / p)^{2}}=\frac{\beta_{x}}{\gamma^{2}} .
$$

As a result, for a high energy $\gamma>\gamma_{t}$, the effect of collapsed velocity distribution dominates over coupling, and the longitudinal IBS can be described by the gas-relaxation formula, independent of the ring lattice.

\section{GAS DIFFUSION EXPRESSED IN BEAM PARAMETERS}

One can rewrite diffusion coefficient

$$
D_{z z} \approx \frac{4 \pi n(Z e)^{4}}{m^{2}} \frac{\Lambda}{\Delta_{\perp}}
$$

in terms of beam parameters. In the laboratory system it becomes:

$$
D_{z z}=\frac{2 r_{i}^{2} c N_{i} \Lambda}{\beta^{3} \gamma^{3} \epsilon_{\perp}^{3 / 2} \beta_{\perp}^{1 / 2} C},
$$

where for the 6-D phase-space density we used $\mu=n /\left(m^{3} \Delta_{\perp}^{2} \Delta_{\|}\right)=N / V_{6}$, with the 6-D volume being $V_{6}=(2 \pi)^{3}(\beta \gamma)^{3} m^{3} \epsilon_{x} \epsilon_{y} \epsilon_{z}$. Here, the formula is written for coasting beam with $C$ being the ring circumference. Note that the normalization factor in velocity space was

already taken into account in the derivation of Eq. 8. One then needs only the 3-D spatial contribution to the normalization coefficient to get from Eq. 8 to 9. Expression given in Eq. 9 was used in the original version of the SimCool code to represent diffusion rate due to the IBS. The longitudinal heating rate for a bunched beam with $C$ being replaced by $2 \sqrt{\pi} \sigma_{s}$ (following the standard definition in the IBS theory, which corresponds to $92 \%$ of a Gaussian beam in longitudinal direction) is then 


$$
\tau_{\|}^{-1}=\frac{1}{\sigma_{p}^{2}} \frac{d \sigma_{p}^{2}}{d t}=\frac{r_{i}^{2} c N_{i} \Lambda}{8 \beta^{3} \gamma^{3} \epsilon_{x}^{3 / 2}\left\langle\beta_{\perp}^{1 / 2}\right\rangle \sqrt{\pi / 2} \sigma_{s} \sigma_{p}^{2}} .
$$

The transverse growth rate can be simply expressed through the longitudinal growth rate as

$$
\tau_{\perp}^{-1}=\frac{\sigma_{p}^{2}}{\epsilon_{x}}\left\langle\frac{D_{x}^{2}+\left(D_{x}^{\prime} \beta_{x}+\alpha_{x} D_{x}\right)^{2}}{\beta_{x}}\right\rangle \tau_{\|}^{-1},
$$

where \langle\rangle is an average value of the ring lattice. For the RHIC lattice the contribution of the term with dispersion derivatives and alpha-function is small (smooth lattice) and can be neglected so that only $\left\langle D_{x}^{2} / \beta_{x}\right\rangle$ may be considered. Recently, the transverse heating rate based on Eq. 11 was implemented in the SimCool code [10] .

\section{APPLICATION TO RHIC}

Based on the discussion in Sections I-III, for high energies at RHIC (when approximation of flat velocity distribution in the beam rest frame becomes valid), simple gas-relaxation

formula gives reasonable approximation for description of the longitudinal heating rate, compared to the involved IBS treatment with complicated dependence on the lattice parameters. This simple formula based on the diffusion coefficient in a gas for a flat velocity distribution was also used in the original version of the SimCool code. The standard IBS formulas can be simplified for high-energy case. For example, Bjorken-Mtingwa (B-M) model can be approximated for a round beam at high-energy as

$$
\tau_{\|}^{-1}=\frac{r_{i}^{2} c N_{i} \Lambda}{8 \beta^{3} \gamma^{3} \epsilon_{x}^{3 / 2}\left\langle\beta_{\perp}^{1 / 2}\right\rangle \sigma_{s} \sigma_{p}^{2}}
$$

The high-energy approximation of B-M agrees very well with the one obtained using the Gas-Relaxation model. This confirms that the main effect in IBS diffusion at high energy is determined by a degree of a collapse of velocity distribution in the beam moving frame of reference. Treatment of the IBS in such a case is then extremely simplified. To describe applicability region of the high-energy approximation one typically introduces parameter $g_{f}$ which describes a degree of a collapse of ion velocity distribution, and is defined as 
$g_{f}=\left(\frac{\Delta_{\|}}{\Delta_{\perp}}\right)^{2}=\frac{\left\langle\beta_{\perp}\right\rangle \sigma_{p}^{2}}{\gamma^{2} \epsilon_{\perp}}$. For typical parameters of Au ions at RHIC store energy $g_{f}$ is in the range $0.1-0.2$ which justifies the use of high-energy approximation treatment of the IBS for simple estimates.

\section{SUMMARY}

In this Note we described the gas-relaxation formula which was used in the original version of the SimCool code to account for the Intrabeam Scattering in RHIC. Such a formula was recently implemented in the BetaCool code and benchmarked vs standard IBS

formulas [11]. This formula is in good agreement with other high-energy approximations of the IBS formulas.

\section{ACKNOWLEDGMENTS}

We would like to thank I. Ben-Zvi, Yu. Eidelman, V. Litvinenko, G. Parzen and G. Trubnikov for many useful discussions in the process of these studies. 


\section{REFERENCES}

[1] I. Ben-Zvi et al., R\&D towards cooling of the RHIC collider, COOL'03 Workshop (Japan, May 2003).

[2] I. Meshkov, A. Sidorin, A. Smirnov and G. Trubnikov, "The BetaCool code" (JINR, Dubna, Russia).

[3] SimCool code was originally written by V.Parkhomchuk et al. (BINP, Russia). We presently use version of the code which is being developed at BNL by I.Ben-Zvi, Yu. Eidelman, A. Fedotov and V. Litvinenko.

[4] A. Sorensen, CERN Acc. School (1987).

[5] C. Bernardini et al., Phys. Rev. Lett., V. 10, p.407 (1963).

[6] H. Bruck and J. LeDuff, Int. Conf. on High-Energy Acc., Frascati (1965).

[7] A. Piwinski, Int. Conf. on High-Energy. Acc., Stanford (1974).

[8] Generalization to include variation of lattice functions was done by Sacherer, Mohl and Piwinski and summarized by M. Martini (1984).

[9] Bjorken and Mtingwa, Particle Acc., V. 13, p.115 (1983).

[10] Yu. Eidelman and A.V. Fedotov, Recent developments in the SimCool code (March 2004), unpublished.

[11] A.V. Fedotov and G. Trubnikov, Benchmarking of the gas-relaxation vs IBS formulas using the BetaCool code (February, 2004), unpublished. 